\title{
Animal Models in Neural Transplantation
} (Report on Session 40.0)

Washington, D.C., July 16, 1992

\author{
Herbert M. Geller \\ Department of Pharmacology, UMDNJ-Robert Wood Johnson Medical School \\ Piscataway, NJ 08854, USA
}

The session on new animal models in transplantation provided a forum for those interested in the use of transplants as a method of understanding critical issues in neuronal development, neural degeneration and localization of function. One major theme was that transplants from a donor with a given behavioral phenotype can restore function following a lesion, and carry the donor phenotype to the host brain. Behavioral restoration was only produced by grafts of specific regions and was correlated with graft survival. An additional theme was that grafts could be used to study the postnatal development of tissue from trisomy-16 mice by grafting the tissue into a normal host. These studies could not be carried out in trisomy-16 animals due to the poor postnatal survival of trisomy-16 mice.

Dr. Michael Lehman $/ 9,10 /$ reviewed his experiments on the restoration of circadian rhythms by neural transplants. Circadian rhythms are daily rhythms of biological functions, including sleep/waking cycles as well as rhythms in hormonal secretion. The clock that drives these rhythms is located in the suprachiasmatic nucleus of the hypothalamus (SCN). Photic input from the retina can entrain the suprachiasmatic nucleus activity to the light/dark cycle; in the absence of such a cycle, such as when animals are kept in constant darkness, animals display an intrinsic rhythm. This rhythm is different in different species, and is even different between different strains of certain species. Both intrinsic and entrained rhythms are abolished when the SCN is destroyed. A major question concerns the nature of the endogenous biological clock. Grafts of embryonic SCN, or grafts of dissociated SCN cells or cultured SCN restore circadian rhythms following SCN destruction. Restoration is accomplished when grafts are placed into the third ventricle without forming connections to the host brain. No recovery was observed with control grafts of other regions, confirming the specificity of the SCN. When grafts are taken from donors that have a different intrinsic rhythm than the host, the restored rhythm is that of the donor. Taken together, this implies that the properties of the graft that restored circadian rhythm are intrinsic to cells within the graft and are not dependent upon synaptic interactions with the host. Whether the rhythm is due to the glia or one of the several subpopulations of peptidergic cells that synthesize vasoactive intestinal polypeptide, neuropeptide $Y$ or vasopressin is an open question. Future studies that use grafts of dissociated cells that have different relative numbers of each of these cell types provide a unique opportunity to evaluate this possibility.

Dr. Federico Bermúdez-Rattoni /1,2/ reviewed work on the ability of fetal brain grafts to enhance recovery of function using behavioral models. Conditioned taste aversion is a very reliable behavioral test: thirsty animals normally drink water, but learn to avoid drinking water containing $\mathrm{LiCl}$, which produces gastric irritation. Following bilateral electrolytic or excitotoxic insular cortex lesions, animals lose conditioned taste aversion; both the ability to acquire and retain the aversion are eliminated. Grafts of embryonic 15 day insular cortical tissue can promote recovery of function after lesion. The recovery is specific to insular cortex, since grafts of tectum, brainstem or occipital cortex did not promote recovery to the extent of insular grafts. The recovery of behavioral function was also accompanied by increases in connectivity between the amygdala and the insular cortex, as well as by a parallel increase in AChE 
and Golgi staining of grafts. The role of NGF as a trophic factor for the cholinergic neurons in the insular grafts was evaluated by adding NGF implants along with the insular tissue. NGF added to the insular grafts promoted more rapid and more extensive recovery than in animals that had received insular grafts alone, whereas NGF alone did not promote recovery. Similar experiments showed that passive inhibitory avoidance, which is also disrupted by insular cortex lesions, can be restored with insular grafts and that NGF addition promotes recovery even further. This suggests that the normal trophic support during development may be necessary to ensure optimal function of transplants from immature tissue.

Dr. Nicole Le Douarin /7,8/ presented experiments that utilized her pioneering system of chick/quail chimeras. These experiments were initially enabled by the fact that the cells of the two different species are distinguishable histologically because interphase nuclei of quail cells contain condensed chromatin; more recently, speciesspecific antibodies that recognize neurites or somata in one species have become available. By replacing one part of the nervous system of chick with quail or vice versa in early embryos, the migration and development of both host and transplanted cells can be followed. The general observation is that tissue from an embryonic graft of dissociated cells will integrate well with the host tissue - animals hatch and behave normally for up to 2 months after birth. Hcwever, an immune reaction eventually develops against the grafted tissue, which results in destruction of the transplanted cells. This destruction is also accompanied by a loss of function, e.g., when spinal cord grafted cells are lost, the host animal becomes paralyzed. These techniques have been applied to the study of cerebellar development. It was thought that the cerebellum came from the mesencephalic vesicle. When the mesencephalic vesicle is transplanted from the quail into the chick, the posterior cerebellum is shown to contain neurons of quail origin, while the medial and anterior cerebellum arises from the chick host. Interestingly, in the anterior part of the cerebellum, where the ventricular epithelium is chick, the Purkinje cells and their surrounding neurons, as well as the neurons of the molecular layer, are of chick origin, while the migrating cells of the interior granular layer are of quail origin. Since cells migrate radially the granule cells must also have a lateral component, which appears to happen as a result of the penetration of the dorsal region of the mesencephalon into the future cerebellum, allowing the formation of an external granular layer of metencephalic origin, while the other cells in the region are of mesencephalic origin. This trajectory appears to overlap with the regions of expression of the engrailed-2 gene. In contrast to xenogenic transplants, there is no rejection in allogenic transplants; this allows the study of grafts from chickens that carry genetic diseases to normal chickens. When tissue is transplanted from genetically epileptic chickens that exhibit photic seizures accompanied by a loss of balance and paralysis, the behavior is observed in host animals that have received grafts of the prosencephalon and the anterior part of the mesencephalon. Interestingly, animals that receive grafts of the prosencephalon alone show an epileptic EEG, but do not exhibit the complete motor seizures, thus localizing the behavioral and EEG manifestations to different regions.

Two presentations concerned the use of the trisomy-16 mouse model of neuronal degeneration $13,5 /$. The trisomy-16 mouse is considered to be an animal model of Down's syndrome, since this mouse contains an extra copy of the region of mouse chromosome 16 that is homologous to human chromosome 21. Among the ten common genes on these chromosomes, Alzheimer's precursor protein (AAP), CuZn superoxide dismutase and the ets- 2 protooncogene are on both segments. Trisomy-16 mice also have phenotypic characteristics similar to Down's syndrome humans, including endocardial cushion defects and similar hematologic and immunologic abnormalities, as well as several developmental abnormalities of the nervous system. Alzheimer's precursor protein (APP) is overexpressed in trisomy-16 mouse brain, even at late gestation. Because trisomy-16 mice die at birth, they are relatively difficult to study. However, transplantation of trisomy-16 tissue into normal

JOURNAL OF NEURAL TRANSPLANTATION \& PLASTICITY 
animals provides an opportunity to evaluate the development of this tissue at later stages.

Dr. David Holtzman /5,6/ focussed on the survival and development of cholinergic neurons when transplanted from normal and trisomy-16 mice into normal animals. The cholinergic neurons that project to the basal forebrain are in the medial septal nucleus, the vertical and horizontal limbs of the diagonal band, and in the nucleus basalis. These neurons degenerate in Alzheimer's disease. Cell suspensions of embryonic basal forebrains from normal or trisomy-16 mice were injected into the hippocampus of 2-4 month old normal mice, with trisomy-16 cells in one hippocampus and control cells in the other. The donor animals had different alleles of Thy-1, which permitted the identification of transplanted tissue. After 6 months, the trisomy16 neurons were atrophied as compared to normals, suggesting that there is an intrinsic difference in their ability to thrive in the normal brain. Given that the hippocampus normally provides trophic support for the cholinergic neuronal population, the question was posed as to whether the increase in levels of NGF that results from removing normal cholinergic input could prevent atrophy. Surprisingly, after making a fimbria-fornix transection, both normal and trisomy-16 neurons were larger. Given that the fimbria-fornix transection alters NGF levels, the question was then addressed whether atrophy could be reversed with NGF. In control animals that did not receive NGF, cholinergic neurons were atrophic and had lower ChAt as compared to controls; however, after NGF infusion both trisomic and control neurons were hypertrophied, with no difference in size between control and trisomy-16 neurons. Thus, while the trisomy-16 neurons degenerate, they retain their response to NGF.

Dr. Christine Hohmann $/ 7,8 /$ addressed the postnatal development of the cerebral cortex of trisomy-16 mice. Chunks of late gestational E15-16 trisomy-16 cortex were grafted into a neonatal host cortex. Both control and trisomy-16 grafts integrated well into the host; typically segregation of cells in different parts of the cortical grafts, as well as invasion of the grafts by AChE-positive fibers were found. Somatostatin (SS) and GAP-43 normally are found in cerebral cortex. There is a normal gradual postnatal increase in SS cells until 3 weeks, then a decline with approaching adulthood. While there was no difference in SS mRNA between trisomy-16 and normal grafts at any age, there was a difference in cell numbers by immunohistochemistry, with more SS cells in trisomy-16 than euploid grafts after four weeks. Interestingly, this difference was due to the fact that trisomy-16 grafts had cell numbers comparable to the normal host, while normal grafts had fewer cells than the host. The pattern of development of GAP-43 mRNA was somewhat similar: very high in neonates, dropping to lower levels in adults. GAP-43 mRNA reduced equally in both types of grafts, but levels in the host were higher than normal.

Both Holtzman and Hohmann investigated expression and synthesis of APP in the grafted trisomy-16 tissue 13,5/. The results from both groups would suggest that there is no consistent overexpression of either APP or $\beta$-amyloid protein in the grafted trisomy-16 tissue. This would suggest that other properties of the host brain may be important for understanding the neural degeneration in the trisomy-16 mouse.

\section{REFERENCES}

1. Bermúdez-Rattoni F, Escobar M, Tapía R, Hiriart $M$. Insular cortical grafts: Factors affecting the recovery of learning. Rest Neurol Neurosci 1992; 4(3): 127-240.

2. Escobar ML, Jiménez N. López-Garcia JC, Tapía R, Bermúdez-Rattoni $F$. Nerve growth factor with insular cortical grafts induces recovery of learning and reestablishes graft choline actyltransferase activity. J Neur Transplant Plast 1993; 4: 167-172.

3. Hohmann CF, Capone GT, Coyle JT. Effects of gene imbalance on amyloid precursor protein [APP] expression in grafts and recipient cortex. Rest Neurol Neurosci 1992; 4(3): 127-240

4. Hohmann CF, Capone G, Oster-Granite ML, Coyle JT. Transplantation of brain tissue from murine trisomy-16 into euploid hosts: effects of gene imbalance on brain development. Prog Brain Res 1990; 82: 203-214.

5. Holtzmann DM, Li Y, Chen KS, Epstein FH, Mobley WC. NGF reverses neuronal atrophy in a mouse model of spontaneous neurodegeneration. Rest Neurol Neurosci 1992; 4(3): 127-240.

6. Holtzman DM, Li YW, DeArmond SJ, McKinley MP, Gage FH, Epstein CJ, Mobley WC. Mouse model of neurodegeneration: atrophy of basal forebrain 
cholinergic neurons in trisomy-16 tranplants. Proc Natl Acad Sci USA 1992; 89(4): 1383-1387.

7. Le Douarin NM. Embryonic neural chimeras to study the development of the nervous system. Rest Neurol Neurosci 1992; 4(3): 127-240.

8. Le Douarin NM. Embryonic neural chimeras in the study of brain development. TINS 1993; 16(2): 64-72.

9. Lehman MN, Doll KZ, Ralph M, Silver R. Restoration of circadian rhythms by neural transplants. Rest Neurol Neurosci 1992; 4(3): 127-240.

10. Ralph MR, Lehman MN. Transplantation: a new tool in the analysis of the mammalian hypothalamic circadian pacemaker. TINS 1991; 14(8): 362-366. 

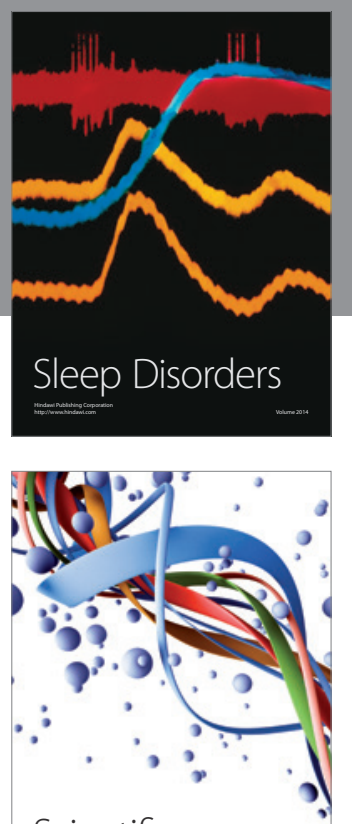

Scientifica
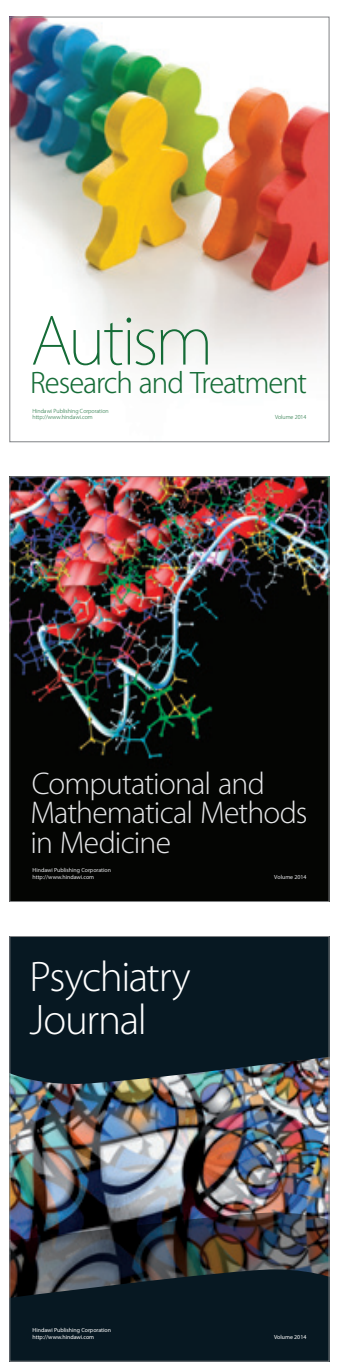
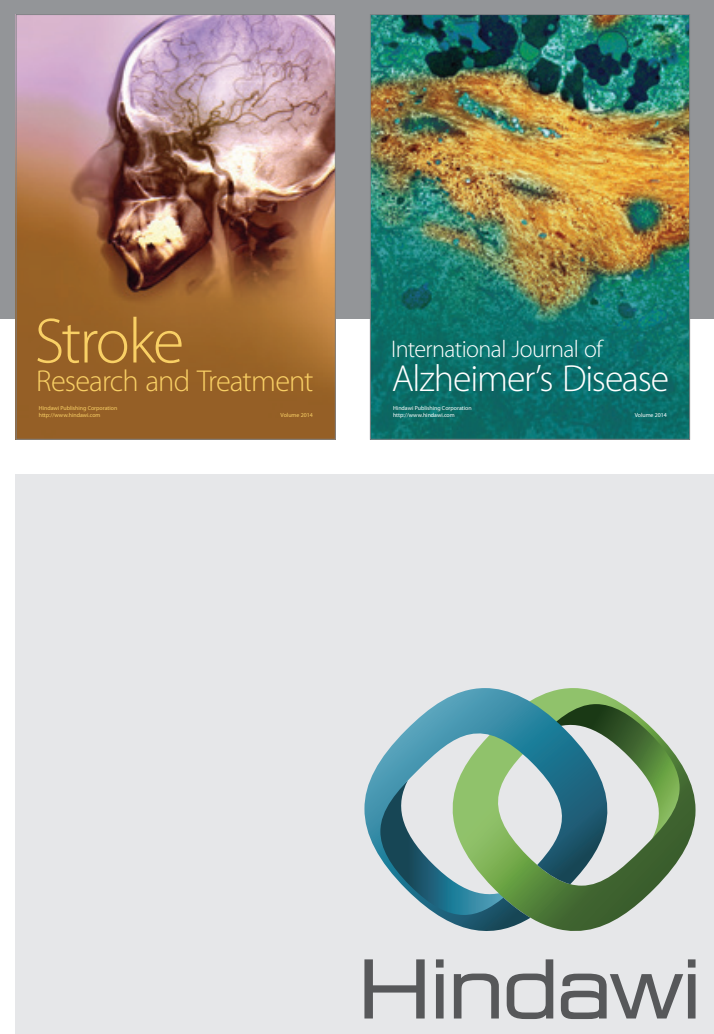

Submit your manuscripts at

http://www.hindawi.com
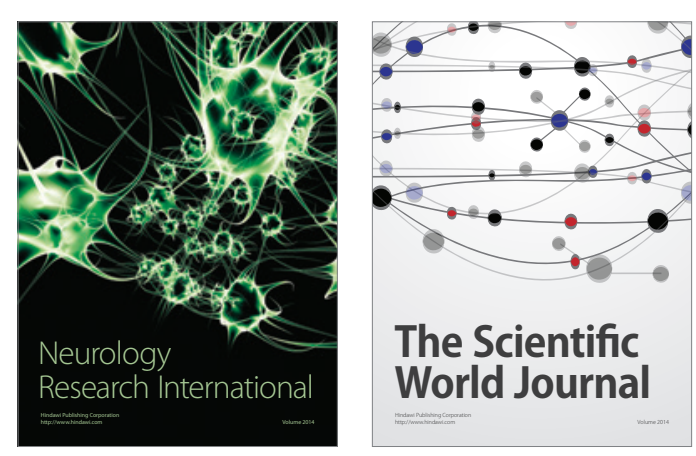

The Scientific World Journal

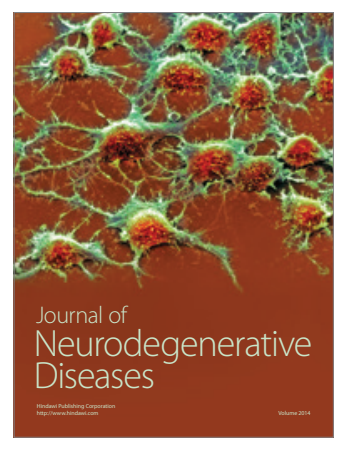

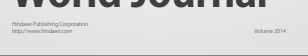

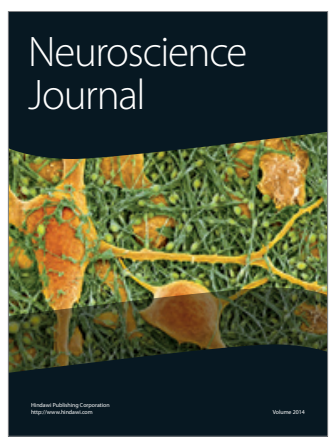

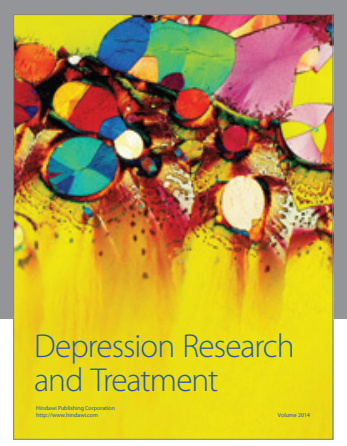
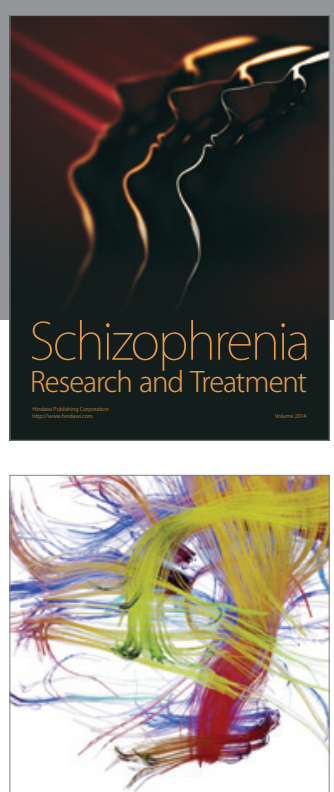

Brain Science

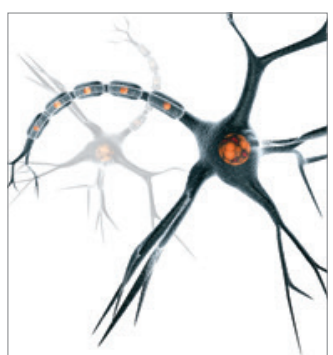

Neural Plasticity
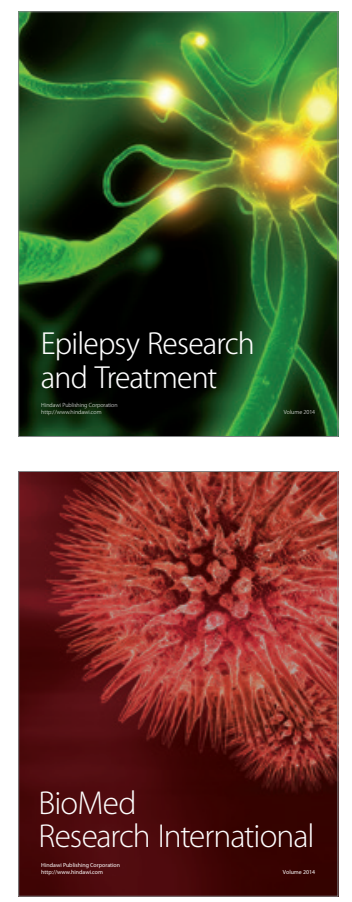

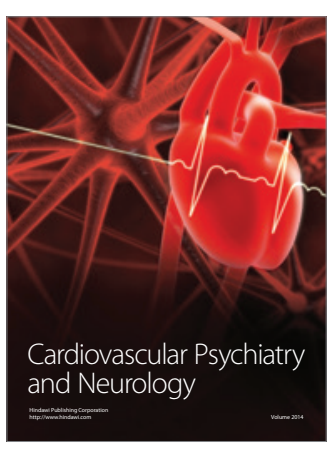

Parkinson's

Disease
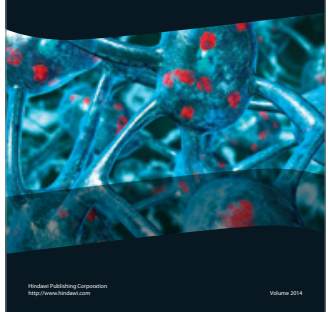